\title{
An Efficient Ring Artifact Reduction Method Based on Projection Data for Micro-CT Images
}

\author{
M. A. Yousuf ${ }^{1}$ and M. Asaduzzaman \\ ${ }^{1}$ Department of Computer Science and Engineering, Mawlana Bhashani Science and Technology \\ University, Santosh, Tangail 1902, Bangladesh
}

Received 19 May 2009, accepted in final revised form 22 November 2009

\begin{abstract}
Ring artifacts are very troublesome in a flat-panel based micro computed tomography (micro-CT) since they might severely degrade visibility of the micro-CT images. Unlike ring artifacts in other types of micro-CTs such as image-intensifier based micro-CT, ring artifacts in a flat-panel detector based micro-CT are hardly removable since the sensitivity of the pixel elements in a flat-panel detector is less uniform than in other types of x-ray detectors. The dependence of the ring artifacts on many imaging conditions, such as tube voltage, detector integration time and phantom size, was first investigated. Based on the observation that the ring artifacts are not imaging-condition-invariant in a flat-panel detector based micro-CT, an efficient ring artifact correction method has been developed based on post-processing. In the filtered sinogram, the ring artifact positions are identified and then the defective lines are corrected in the original projection data before the filtered back-projection. Experimental results on capacitor phantom, contrast phantom and bone images verify the efficacy of the proposed method.
\end{abstract}

Keywords: Micro-CT; Ring artifact correction; Flat-panel detector; Filtered back-projection; Small animal imaging.

(C) 2010 JSR Publications. ISSN: 2070-0237 (Print); 2070-0245 (Online). All rights reserved.

DOI: $10.3329 /$ jsr.v2i1.2645

J. Sci. Res. 2 (1), 37-45 (2010)

\section{Introduction}

Ring artifact correction is not an important issue in medical CTs or charge-coupled-device (CCD) based micro-CTs since ring artifacts can be easily reduced to an acceptable level by the conventional white-field correction. But in flat-panel-detector (FPD) based micro-CTs, ring artifacts are hardly removed since the sensitivity of pixel elements in flat-panel detectors is less uniform than in other type of detectors and it often drifts depending on the imaging condition. Although the ring artifacts are in many cases just an annoyance, it is often a serious problem in small animal imaging using a micro-CT in which image SNRs

\footnotetext{
${ }^{1}$ Corresponding author: usuf672@yahoo.com
} 
are often very low. So, reduction of ring artifacts is essential in small animal imaging. Despite the ring artifact problems, flat-panel detectors are attractive for micro-CTs since they have many advantages over other types of detectors. The flat-panel-detectors are capable of large-area detection with thin structure, and they no geometrical distortions [1, 2]. Since the flat-panel-detector technology is fast growing by the needs of mass production, availability and low cost are other merits in a micro-CT. Ring artifacts are caused by imperfect detector pixel elements as well as by defects or impurities in the scintillator crystals [3, 4]. Ring artifacts can also be generated from higher energy harmonics in the incident beam [5,6]. In reconstructed images, ring artifacts appear as narrow rings or wide bands depending on how many detectors have the spurious behavior. Full rings are generated only if a 360 degree data acquisition is used and half rings with 180 degree acquisition [7].

Other than by the white-field correction method, ring artifacts can also be reduced by applying various image processing techniques on reconstructed images or projection data, so called sinogram [8-10]. The techniques have pros and cons in terms of image quality and processing time. In this paper, we introduce an efficient ring artifact removal algorithm for FPD based micro-CTs. It is known that ring artifacts in FPD based micro-CTs are hardly removed by the conventional white field correction method due to drifts in detector element sensitivity in between white-field calibrations $[5,11,12]$. We observed that ring artifact intensity and position in a FPD based micro-CT are affected by various imaging parameters such as the tube voltage, the detector integration time and the x-ray path length. Especially, the dependence on the path length makes the white field correction method less useful for ring artifact removal in a FPD based micro-CT since the scanning motion in the micro-CT always changes the path length over the viewing angle. Based on the observation, we devised a detection method of defective lines on the projection data. Once the defective lines detected, the defective lines can be corrected by interpolation. Experimental results on small animal images are presented.

\section{Formation of Ring Artifacts}

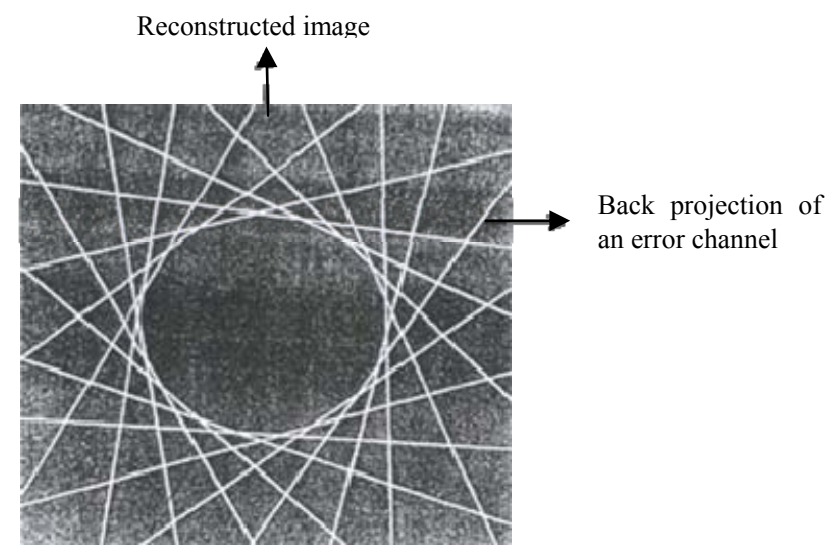

Fig. 1. Illustration of formation of ring artifact. 
Ring artifacts are mainly a third-generation CT phenomenon. They are caused by errors in a single or multiple channels over an extended range of views. An error persistent over an extended view range produces a set of lines with a fixed distance to the iso-center. The tail portions of the lines are canceled, and a ring is formed when the neighboring views are closely placed. The production of ring artifacts is shown in Fig. 1.

\section{Methods}

\subsection{Observation of ring artifacts in a FPD based micro-CT}

We first observed ring artifact dependence on imaging parameters in the images taken with a homemade micro-CT. The homemade micro-CT consists of a flat-panel detector (C7943CP-02, Hamamatsu, Japan) and a micro focus x-ray tube (L8121-01, Hamamatsu, Japan). The micro-focus $x$-ray source is a sealed tube with a fixed tungsten anode having an angle of $25^{\circ}$ against the electron beam and with a $200 \mu \mathrm{m}$-thick beryllium exit window. The emitted x-ray beam span angle is about $43^{\circ}$. The source has a variable focal spot size from $5 \mu \mathrm{m}$ to $50 \mu \mathrm{m}$ depending on the applied tube power (Watt or $\mathrm{kVp} \times \mathrm{mA}$ ). The maximum tube voltage and tube current are $150 \mathrm{kVp}$ and $0.5 \mathrm{~mA}$, respectively. The micro-focus $\mathrm{x}$-ray source has been operated in a continuous mode with an Al filter with the thickness of $1 \mathrm{~mm}$. The flat-panel detector consists of a $1248 \times 1248$ active matrix of transistors and photodiodes with a pixel pitch of $100 \mu \mathrm{m}$, and a CsI:TI scintillator. The CsI:TI has a columnar structure with a typical diameter of about $10 \mu \mathrm{m}$ and the thickness of $200 \mu \mathrm{m}$. A computer-controlled rotating system was adopted in the object holder to achieve a cone-beam mode scan in the micro-CT. The precision of the rotational motion is $0.083^{\circ}$ which allows the number of views larger than 4000 .

We have taken micro-CT images of cylindrical uniform phantoms, $4 \mathrm{~cm}$ and $8 \mathrm{~cm}$ in diameter, with varying tube voltages and detector integration times. To visualize the ring artifacts more clearly, we also reconstructed images using the defective lines only. The defective lines were identified by the method described hereinafter. Fig. 2 shows the ring artifact images of $8 \mathrm{~cm}$ phantom taken with tube voltages of $40 \mathrm{kV}, 60 \mathrm{kV}$ and $100 \mathrm{kV}$ respectively. Tube currents are $400 \mu \mathrm{A}, 110 \mu \mathrm{A}$, and $40 \mu \mathrm{A}$, respectively, and the detector integration time is 0.5 seconds.

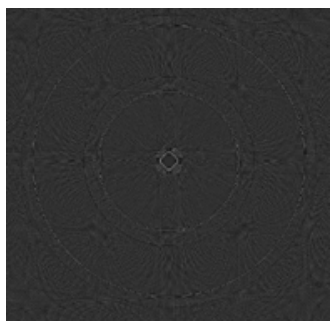

(a)

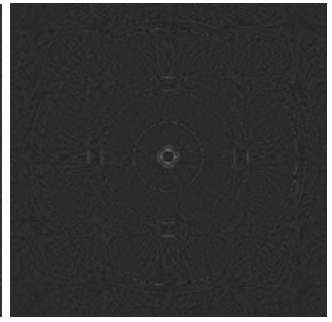

(b)

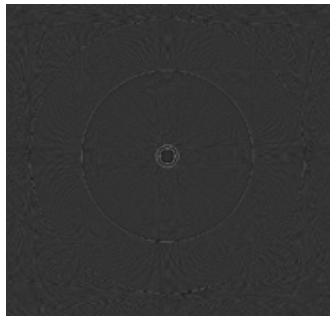

(c)

Fig. 2. Ring artifact images of $8 \mathrm{~cm}$ uniform phantom taken with tube voltages of (a) $40 \mathrm{kV}$, (b) $60 \mathrm{kV}$ and (c) $100 \mathrm{kV}$, respectively. 


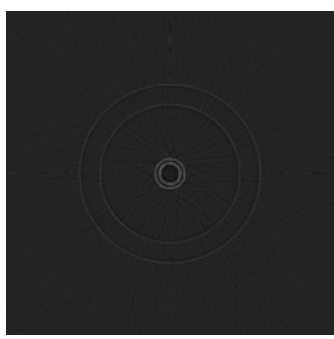

(a)

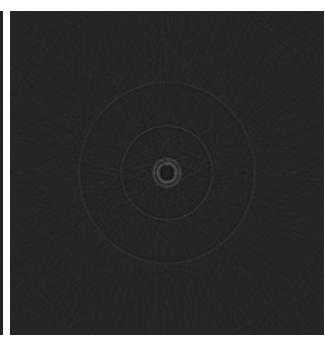

(b)

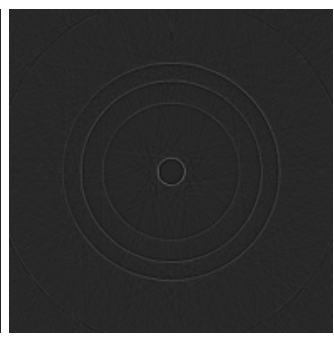

(c)

Fig. 3. Ring artifact images of $4 \mathrm{~cm}$ uniform phantom taken with tube voltages of (a) $40 \mathrm{kV}$, (b) $60 \mathrm{kV}$ and (c) $100 \mathrm{kV}$, respectively.

Fig. 3 shows the ring artifact images of $4 \mathrm{~cm}$ phantom taken with tube voltages of $40 \mathrm{kV}$, $60 \mathrm{kV}$ and $100 \mathrm{kV}$ respectively. Tube currents are $400 \mu \mathrm{A}, 110 \mu \mathrm{A}$, and $40 \mu \mathrm{A}$, respectively, and the detector integration time is 0.5 seconds. It can be observed that the ring artifact patterns are dependent not only on the tube voltages but also on the phantom size. It seems that the dependence on the phantom size is caused by the fact that the transmitted x-ray spectra are different for the two phantoms due to the different path length, hence, different beam hardening effects. Fig. 4 shows the ring artifact images obtained with the detector integration time of $0.5 \mathrm{~s}$ and $1.0 \mathrm{~s}$ at the tube voltages of 40,60 , and $100 \mathrm{kV}$. The ring artifact patterns are also dependent on the detector integration time.
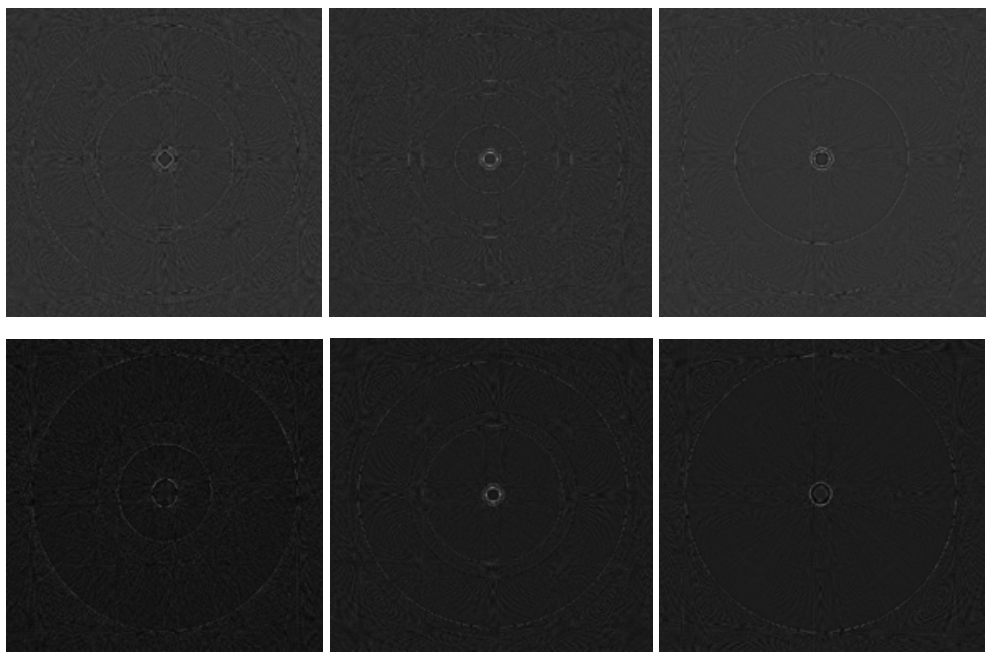

(a)

(b)

(c)

Fig. 4. Ring artifact images of $8 \mathrm{~cm}$ uniform phantom taken with detector integration time of $0.5 \mathrm{~s}$ (top) and $1.0 \mathrm{~s}$ (bottom), respectively, at the tube voltages of (a) $40 \mathrm{kV}$, (b) $60 \mathrm{kV}$ and (c) $100 \mathrm{kV}$, respectively. 


\subsection{Identification and correction of ring artifacts}

With the observation that the ring artifacts in the micro-CT images are dependent on various imaging parameters, we have developed a ring artifact correction method based on post-processing. We first tried to identify the defective lines on the projection data or so called sinogram. A sinogram is formed by stacking all projection data of different views [13]. The complete set of projection data over $M$ projections with $N$ rays per projection can be represented as a two dimensional array of dimension $M \times N[14,15]$. Each row in two dimensional sinogram represents the data for a single projection. Each column indicates the information recorded by a particular detector element for each projection [8]. So if there is any defective element in detector, it will corrupt a single column in the sinogram data. So before correcting line artifacts, we need to find out these corrupted columns (line artifacts in vertical position). The steps of the defective line identification are as follows:

(i) Apply one dimensional horizontal median filter on the filtered sinogram $\tilde{P}(s, \theta)$ in the $s$-direction. The projection data filtered with the $|\omega|$ kernel has more discernible defective lines than the original projection data $P(s, \theta)$. However, the filtered projection data often show very vague defective lines if the projection data have been corrected by the white-field correction. Since the defective pixels appear as delta-like peaks in the $s$-direction, most of the defective lines can be removed in the median-filtered projection data, $\bar{P}(s, \theta)$.

(ii) Subtract the median-filtered projection data from the original filtered projection data, $D(s, \theta)=\tilde{P}(s, \theta)-\bar{P}(s, \theta)$. This subtraction yields only the defective lines.

(iii) Integrate $D(s, \theta)$ along the $\theta$-direction to get $\bar{D}(s)=\int_{0}^{2 \pi} D(s, \theta) d \theta$. The defective lines are more discernible in $\bar{D}(s)$ than in $D(s, \theta)$ since the defective line effects are accumulated in the integration.

(iv) Identify the defective line positions by applying a threshold $T$ to $\bar{D}(s)$. The pixels having greater values than the threshold are identified as defective lines.

The threshold value $T$ in step IV has been determined from the standard deviation of $\bar{D}(s)$. In this work, $T$ has been chosen from 2 times to 3 times the standard deviation.

With the defective lines identified, we applied two dimensional median filter of $3 \times 5$ size to the pixel elements on the defective lines in the projection data $P(s, \theta)$ for defective lines correction. The size of the median filter depends on the severity of defective lines in the projection or sonogram data. After defective line corrections, we reconstructed micro-CT images using the filtered back-projection method.

\section{Results and Discussions}

Ring artifacts correction has been performed on the sinogram data using the proposed method. To test our method we took different kinds of phantom like capacitor, contrast phantom and reconstructed image of femur region of a rat. Fig. 5(a) shows the sinogram of capacitor phantom which is corrupted by defective lines due to the defective pixel of the 
flat-panel detector and Fig. 5(b) shows the line artifacts free sinogram after applying our proposed correction method.

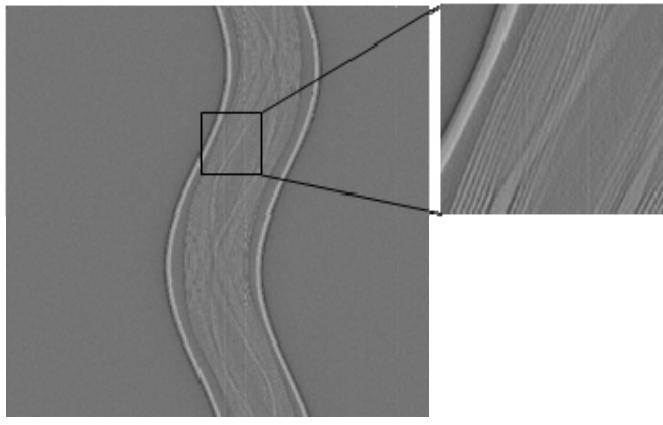

(a)

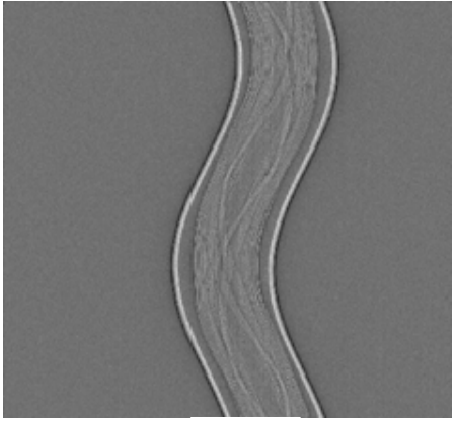

(b)

Fig. .5. Sinogram of capacitor phantom. Sinogram consists of all 450 measured projection data; (a) Sinogram exposes line artifacts that are responsible for ring artifacts. The insert in the top right corner is the magnification of area surrounded by black box which emphasizes line artifacts. (b) Sinogram after removing line artifacts by applying our proposed correction method.

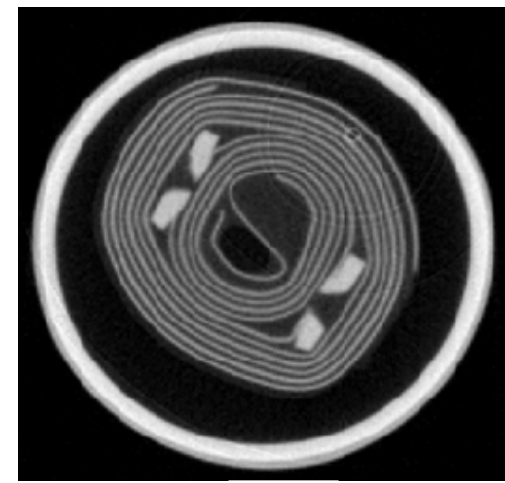

(a)

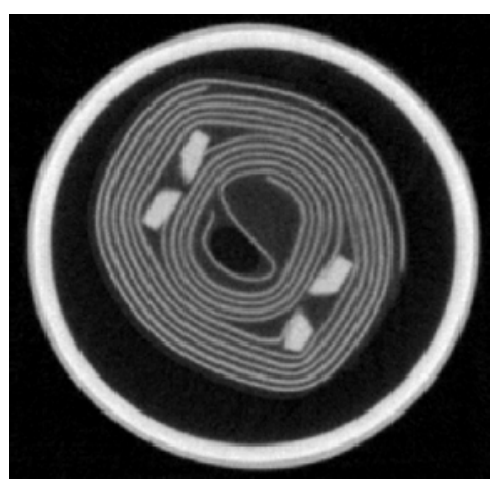

(b)

Fig. 6. Cross-sectional reconstructed image of capacitor phantom. (a) Before ring artifacts corrections. The image is reconstructed from the sinogram of Fig. 5(a). In this image ring artifacts are clearly visible. (b) After ring artifacts correction. The image is reconstructed from the sinogram of Fig. 5(b).

Fig. 6(a) illustrates the cross-sectional image of the capacitor which is reconstructed from sinogram with line artifacts of Fig. 5(a). After reconstruction, line artifacts become ring artifacts which are clearly visible in the reconstructed image in Fig. 6(a). Fig. 6(b) presents the corresponding ring artifacts free correct tomographic image which is reconstructed from line artifact free correct sinogram of Fig. 5(b). 
Contrast phantom image is also used for correcting ring artifacts. Contrast phantom consists of six inserts whose physical densities are very similar to that of water. The materials of six inserts are acrylic, polystyrene, plycarbonate, plastic water, nylon, and polyethylene. The six inserts with $5 \mathrm{~mm}$ diameter were immersed in a water bath made of a $40 \mathrm{~mm}$ diameter acryl cylinder. The inserts were made of commercial electron density phantoms. Fig. 7(a) shows the cross-sectional image of the contrast phantom with ring artifacts at the central region and also some ring artifacts in peripheral region. Fig. 7(b) shows the correct reconstructed image after removing the ring artifacts. As can be noticed from Fig. 7(b), the ring artifacts have been reduced considerably.

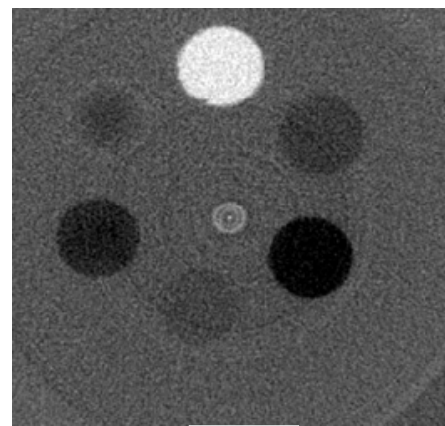

(a)

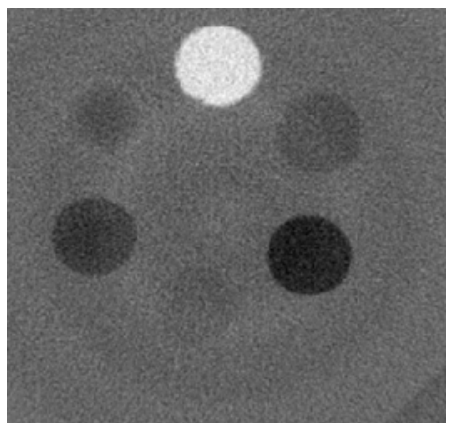

(b)

Fig. 7. Cross-sectional images of the contrast phantom; (a) before and (b) after the ring artifacts correction.

To test our proposed method we also took images of the femur region of a rat. Fig. 8 shows the reconstructed images of the femur region with and without ring artifacts. In Fig $8(a)$, the scan data are corrupted by ring artifacts. Ring artifacts are visible in the central region. Fig 8(b) shows the corrected images after removing ring artifacts. From these figures, it is clear that ring artifacts are significantly reduced.

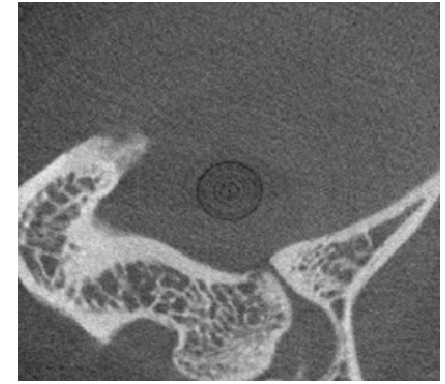

(a)

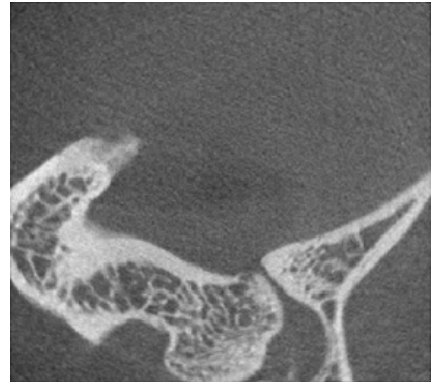

(b)

Fig. 8. Reconstructed images of the femur region of a rat. (a) Image showing ring artifacts, (b) Corresponding ring artifacts corrected image. 
In all the above mentioned cases, 450 projections are taken to form complete set of projection data or sinogram. The matrix size of all above mentioned reconstructed image is $512 \times 512$. The ring artifact correction approach outlined in this paper for sinogram can also be used to correct ring artifacts on reconstructed images. Although it is preferable and also more effective to correct sinogram data, only the image data are available in many situations. That is in many cases only reconstructed images are available not the sinogram or projection data. In that case ring artifacts need to be corrected in reconstructed image. But if sinogram or projection data are available, it is more effective to correct sinogram data. In ref. [9], at first raw input image of size $\mathrm{P} \times \mathrm{Q}$ showing ring artifacts is transformed into polar coordinates. Next the line pattern in polar coordinate image is reduced by detecting this pattern from homogeneous row segments in polar coordinate image. To do this a window of fixed size id slid across the polar coordinate image. For each row of window, the signal variance is computed. Then a threshold value is calculated for identifying row segments as homogeneous or inhomogeneous. If signal variance is smaller than a calculated threshold, this row is subtracted by its mean value (normalize) and stacked as a row into a temporary artifact matrix. The final size of the artifact matrix is given by the collection of all rows that meet the homogeneity criterion. That is all the rows whose variances are smaller than the specified threshold value. Then, for each column of artifact matrix, the median value is computed. This results in an artifact template vector. For each window position, the artifact template is stored into a final artifact template row of size $1 \times \mathrm{Q}$ if the number of rows that meet the homogeneity criterion in that particular window position, is maximal. Finally, the artifact template vector is subtracted from each row of the polar coordinate image.

To remove ring artifacts from the reconstructed images, the input images need to be transformed into the polar coordinates [9]. In this case, two-fold interpolation scheme is needed. One is needed while transforming from the Cartesian coordinates image into the polar coordinates image and the other interpolation is needed while transforming back from the polar coordinates into the Cartesian coordinates. So it is very difficult to preserve the image resolution in all cases. In addition in ref. [9], the artifacts are corrected in the polar coordinates without any distinction of pixels with and without artifacts. That is, they have changed all pixel values including the pixel values without artifacts. As a result they were not able to keep original image as it was before ring artifacts correction.

In our method, we do not need to use any interpolation scheme for ring artifacts correction. So the main advantage of the proposed method is that no interpolation scheme is required for correcting ring artifacts in sinogram data. Besides we have changed the pixel values of defective lines only and kept other pixel values as it was before ring artifacts correction. As no interpolation scheme is used and only the pixel values of defective lines are changed to correct ring artifacts, the image resolution is hardly affected. Furthermore, as only defective lines are corrected in our proposed method, this method is faster than the correction method on reconstructed image mentioned in ref. [9].

\section{Conclusions}

In this paper an efficient method is introduced to remove ring artifacts from micro-CT images. The method has been evaluated on a capacitor phantom, a contrast phantom and 
femur region of a rat. Experimental results have shown that ring artifacts can be removed efficiently without noticeable loss of spatial resolution.

\section{Acknowledgments}

We are thankful to all members of Medical Imaging System Lab, Department of Biomedical Engineering, Kyung Hee University, Korea.

\section{References}

1. H. J. Kim, H. K. Kim, G. Cho, and J. Choi, Nucl. Instrum. Methods Phys. Res. A 505, 1155 (2003).

2. R. Ning, B. Chen, R. Yu, D. Conover, X. Tang, and Y. Ning, J. IEEE Trans. Med. Imaging 19 (9), 949 (2000).

3. C. Antoine, P. Nygard, W. O. Gregersen, R. Holmstad, T. Weitkamp, and C. Rau, Nucl. Instrum. Methods Phys. Res. A, 490, 392 (2002). doi:10.1016/S0168-9002(02)01003-3

4. M. Axelsson, S. Svensson, and G. Borgefors, Reduction of Ring Artifacts in High Resolution X-Ray Microtomography Images in Proc. DAGM 417461 (2006).

5. X. Tang, R. Ning, R. Yu, and D. Conover, J. Med. Phys. 28, 812 (2001).

6. R. A. Ketcham and W. D. Carlson Acquisition, Computers \& Geoscience 27, 381 (2001). doi:10.1016/S0098-3004(00)00116-3

7. R. A. Ketcham, New algorithms for ring artifact removal in Proc. SPIE. 6318, 63180O1 (2006).

8. C. Raven, Review of Scientific Instruments 69, 2978 (1998). doi:10.1063/1.1149043

9. J. Sijbers, and, A. Postnov, Phys. Med. Biol. 49, 247 (2004). doi:10.1088/0031-9155/49/14/N06

10. M. Boin and A. Haibel, Opt. Express 12071 (2006). doi:10.1364/OE.14.012071

11. P. Cloetens, Contribution to Phase Contrast Imaging, Reconstruction and Tomography with Hard Synchrotron Radiation - Principles, Implementation and Applications, PhD thesis, Vrije Universiteit Brussel (1999).

12. U. Kumar and G. S. Ramakrishna, Applied Radiation and Isotopes 57, 543 (2002). doi:10.1016/S0969-8043(02)00134-3

13. J. Hsieh, Computed tomography: principles, design, artifacts and recent advances. Washington: SPIE Press 167 (2003).

14. G. T. Herman, Med. Phys. 9, 446 (1982).

15. A. K. Jain, Fundamentals of Digital Image Processing (Englewood Cliffs, Prentice Hall, New Jersey, 1989) pp. 431-475. 